\title{
Estandarización en la cerámica prehispánica de El Caño, Panamá: especialización, productividad y consumo
}

\author{
Carlos MaYo \\ Centro de Investigaciones Arqueológicas del Istmo, Fundación El Caño, Panamá \\ carlosmayo@fundacionelcano.org
}

Recibido: 26 de noviembre de 2013

Aceptado: 13 de noviembre de 2014

\begin{abstract}
RESUMEN
La cerámica encontrada en las tumbas de El Caño comparte el mismo estilo que la producida en las llanuras aluviales de la región en los siglos comprendidos entre el 700 y 1000 d.C. Los datos de los coeficientes de variabilidad de las diferentes formas analizadas evidencian distintos valores de estandarización en la producción de la vajilla polícroma y la no polícroma. Los contextos funerarios excavados recientemente en el Caño indican además que la producción de cerámica polícroma era controlada por una élite. Este control sobre la producción de ciertos bienes sugiere que estos fueron clave en el desarrollo de las jefaturas en el istmo de Panamá y marca la fase de esplendor de la cultura coclesana.
\end{abstract}

Palabras clave: Estandarización, especialización artesanal, cerámica prehispánica, Coclé, Arqueología de Panamá.

\section{Standardization in the Pre-Columbian Pottery from El Caño, Panama: Specialization, Productivity and Consumption}

\begin{abstract}
The pottery found in the burials of El Cano is uniform in style to these made in the coclesanos valleys between 700 and $1000 \mathrm{AD}$. The coefficient of variability of the different pottery forms, evidence diverse standardizations values for polychrome and non-polychrome ceramics. Moreover, data of funerary contexts from the Cano recently excavated, suggest that elite has controlled ceramic production. This control over the production of certain goods reveals that these were important in the support or proper operational of the chiefdoms in Panama and mark the phase of splendour of this culture.
\end{abstract}

Key words: Standardization, Craft Specialization, Prehispanic Pottery, Coclé, Panama Archaeology.

Sumario: 1. Introducción. 2. Descripción y ocupación de sitio El Caño 3. Contexto regional: producción y consumo de la cerámica polícroma en la región cultural de Gran Coclé y territorios adyacentes. 4. Método. 5. Materiales. 6. Análisis. 7. Resultados. 8. Control económico de la producción de cerámica en Coclé. 9. Discusión. 10. Referencias bibliográficas.

\section{Introducción}

La cerámica objeto de análisis en este artículo fue extraída en el yacimiento de El Caño y guarda un gran parentesco con la encontrada en las provincias centrales, en especial con las de Sitio Conte (Lothrop 1942), yacimiento clave para el estudio de las sociedades complejas en Panamá. Es una de las cerámicas más sofisticadas producida en la América Prehispánica por su luminosidad, cromatismo, riqueza temática y estilística. Lo que aquí se presenta, sin embargo, no es un estudio sobre el virtuosismo y desarrollo de su arte pictórico (Labbé 1995) o sobre la simbología de sus diseños (Helms 1995, 2000) ni sobre otros temas ampliamente tratados con anterioridad por 
otros autores (Lothrop 1976; Cooke 1985, 2011; Mayo 2006), sino un análisis sobre el grado de estandarización en la cerámica de la sociedad coclé, el cual tiene implicaciones teóricas de cómo se organizaba la producción de la misma entre los años 700-1000 d.C.

En los estudios cerámicos, la estandarización es vista como una herramienta que permite elaborar hipótesis sobre su sistema productivo dependiendo del grado de uniformidad de las piezas que son objeto de estudio. Estas hipótesis suelen estar ligadas al concepto de especialización y a los procesos de producción y son sustentadas a través de estudios etnográficos (Hagstrum 1988; Longacre et al. 1988; Costin 2000). Una variabilidad baja o, lo que es lo mismo, una estandarización elevada, se interpreta como una manufactura especializada organizada en torno a una o pocas unidades de producción (Rice 1981; Costin 1991, 2001). Por el contrario, una variabilidad alta indica producciones diversificadas en ambientes domésticos (Benco 1988).

La uniformidad de las vasijas también es signo de la destreza y experiencia del artesano (Longacre 1999). Por razonamiento lógico, estas habilidades se incrementan con la edad (Kramer 1985) y con las horas que el artesano destina a la actividad, ya sea ésta a tiempo parcial o completo (Rice 1981) con un evidente aumento de la productividad en la manufactura. La productividad es otra característica que se desprende de los análisis de estandarización (Kramer 1985; Rice 1991). La organización del trabajo en uno o pocos centros de producción, con artesanos trabajando a tiempo completo, aumenta la manufactura de bienes y reduce la variabilidad de artefactos (Costin y Hagstrum 1995) por lo que, dependiendo del grado de homogeneidad de las colecciones, podemos aproximarnos al volumen de producción (Roux 2003).

Para saber si la cerámica objeto de este estudio es una colección estandarizada, se analizaron aquellos conjuntos o grupos cerámicos presumiblemente coetáneos. Estos fueron comparados con resultados de estudios etnográficos llevados a cabo en India, Filipinas, España y México (Longacre et al. 1988; Roux 2003; Arnold et al. 2010), en los que se infieren datos sobre producciones a gran escala realizados en talleres por artesanos especializados a tiempo completo y, en otros contextos de producción, por artesanos a tiempo parcial en producciones domésticas.

Estos análisis se realizarán para conocer los diferentes niveles de estandarización en la producción de cerámica, ya sea ésta de carácter ritual (vajilla polícroma) o utilitaria (vajilla roja y roja sobre crema). A su vez, se contrastarán los datos arqueológicos de diferentes contextos arqueológicos de la región para conocer la distribución geográfica y el consumo de cerámica polícroma entre la élite y los entierros más humildes.

\section{Descripción y ocupación del sitio El Caño}

El yacimiento funerario de El Caño se encuentra en la llanura aluvial de Río Grande a unos $20 \mathrm{~km}$ de su desembocadura (Figura 1). Es un sitio multicomponente con estructuras de piedra (alineamientos «columnarios»), esculturas y montículos de tierra antrópicos. Hasta las recientes excavaciones, El Caño (Mayo y Mayo 2013) era comúnmente aceptado como un centro donde se desarrollaban actividades sociales 


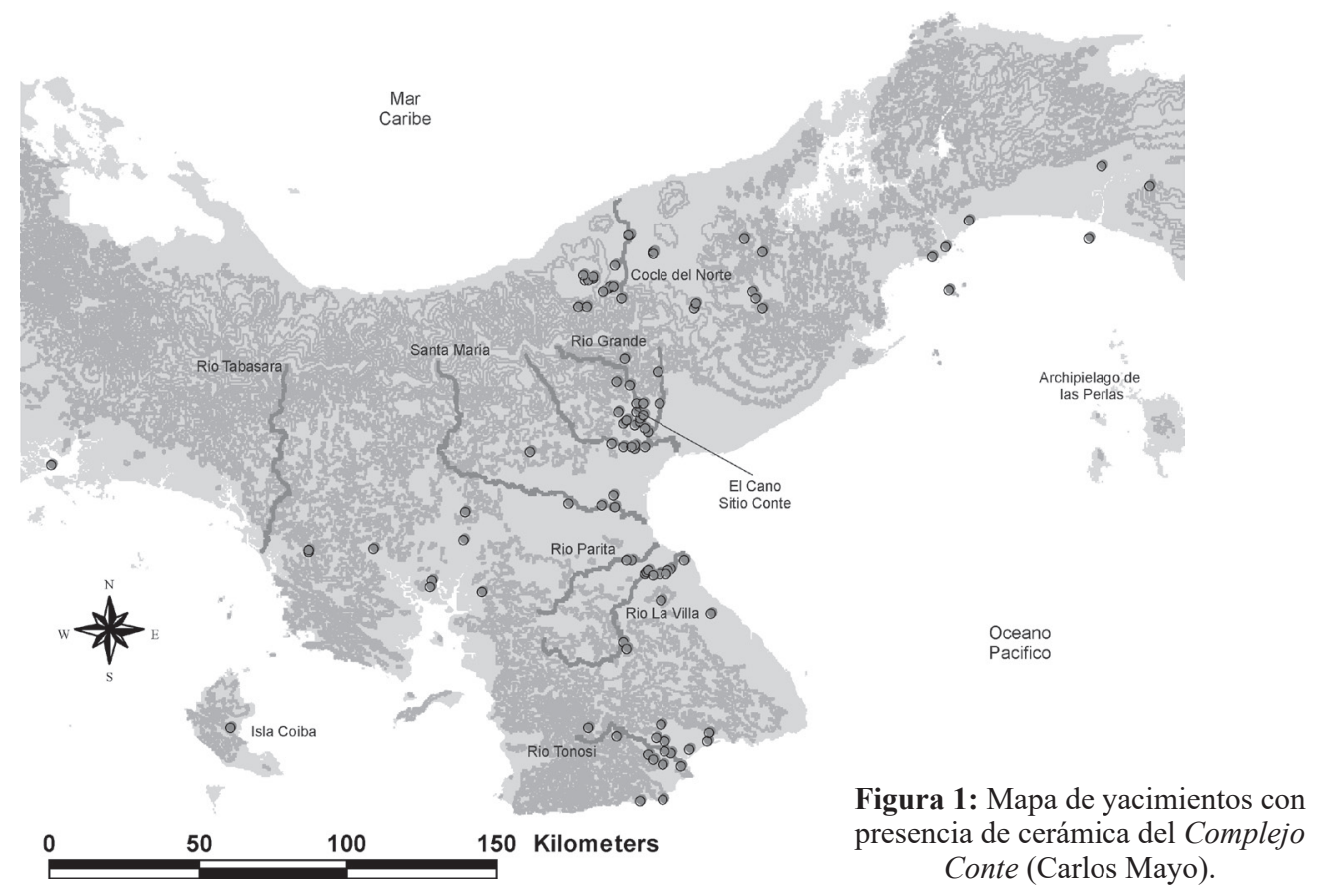

de carácter ritual (Fitgerald 1992), vinculadas al yacimiento funerario de Sitio Conte, siendo Cerrezuela el mayor yacimiento habitacional de las llanuras. Sin embargo, ahora en El Caño se han descubierto tumbas de gente rica y poderosa, al igual que en Sitio Conte, por lo que se reabre la discusión sobre los usos y funciones de estos yacimientos.

El Caño se excavó por primera vez en la década de 1920 (Verrill 1927). El material más informativo en estos trabajos fue un conjunto de esculturas cuya importancia radica en su marcado carácter narrativo y podrían estar relacionadas con las actividades que se desarrollaban en este lugar. A partir de investigaciones recientes (Mayo et al. 2010) las vinculamos con los areitos descritos en las crónicas españolas (Fernández de Oviedo 1853; Andagoya 1865) y que relacionan directamente este conjunto de esculturas con las actividades funerarias desarrolladas en el sitio. Lastimosamente cualquier lectura de este conjunto escultórico es sumamente difícil, ya que no se ha podido reconstruir su ubicación original y la relación de éstas con el grupo se ha perdido.

Intervenciones posteriores, ponen de manifiesto los distintos periodos de ocupación del yacimiento. En las proximidades del alineamiento principal de monolitos se encontraron tumbas múltiples que deben adscribirse al periodo Cerámico Tardío A (Zelsman 1959; Doyle 1960). Con posterioridad, trabajos del ingenio vecino rompieron algunos depósitos funerarios en la zona de montículos, lo que provocó una intervención de rescate (Cooke 1976a). En esta intervención se encontró cerámica del periodo de contacto adscrita al Cerámico Tardío D y E (1500-1520). En los años 80 se excavó uno de los montículos (Lleras y Barillas 1980) y se encontraron distintos 


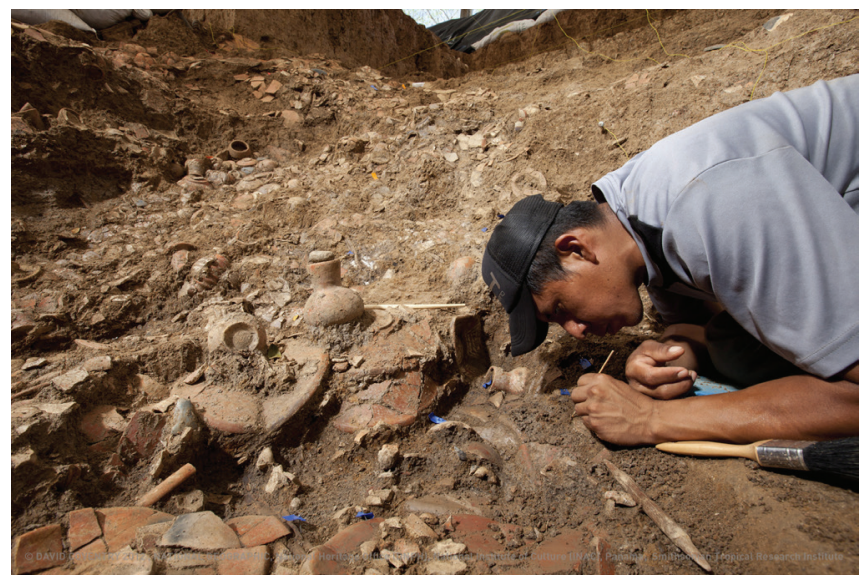

Figura 2: Proceso de excavación de los depósitos funerarios de la Tumba 2, El Caño (Fotografía de David Coventry, National Geographic Society).

episodios funerarios del Cerámico Tardío A y B (700-1100 d.C.) bajo un denso estrato con cerámica del Cerámico Tardío D y E (1300-1520), además de algunos pisos de ocupación y cerámica semicocida. Estos pisos de ocupación, con restos de cerámica y suelos quemados, pudieron estar vinculados a las tumbas encontradas en los montículos más que a zonas de viviendas, algo que ya se señala en los estudios posteriores de la estratigrafía del Montículo 3 y 4 hecha por el arqueólogo Carlos Fiztgerald (1992).

En el año 2005, el «Proyecto Arqueológico El Caño» inicia sus trabajos en el sitio ante la seguridad de que permanecían todavía ocultos depósitos funerarios y estructuras antiguas. Se hicieron prospecciones geofísicas (Mojica et al. 2007) y microtopografias del terreno para identificar posibles estructuras. Concluidos estos trabajos en el año 2008 se comenzaron las excavaciones (Figura 2) que actualmente están en progreso (Mayo y Mayo 2013). Desde entonces se han excavado cuatro depósitos funerarios de personas de alto estatus que han proporcionado las primeras fechas absolutas del yacimiento (780-1020 d.C.), y que coinciden con las fechas del periodo Cerámico Tardío A y B (Mayo y Mayo 2013). En los rellenos de dichas tumbas se ha hallado asimismo cerámica que debería adscribirse a finales del Cerámico Medio.

Por lo tanto, existe una ocupación del yacimiento, con un periodo de abandono, desde finales del Cerámico Medio hasta la Conquista española. Se desconoce la función del sitio a finales del Cerámico Medio, ya que sólo se han encontrado muestras muy fragmentadas en los rellenos de las tumbas excavadas. A comienzos del Cerámico Tardío (700-1100 d.C.) el sitio fue usado como lugar de entierro, tanto de personas humildes (zona de montículos), como de personas de élite. Se cree que el conjunto de esculturas, los llamados alineamientos «columnarios», son coetáneos con estos entierros y desempeñaban un papel importante en las actividades funerarias desarrolladas en el sitio (Fitzgerald 1992; Mayo et al. 2010). La ausencia de cerámica del periodo Cerámico Tardío C (1100-1300 d.C.) se interpreta como un periodo de abandono que coincide con una crisis generalizada en todo el continente americano. El Caño vuelve a ocuparse, como lugar de entierro, durante el complejo El Hatillo y Mendoza (13001520 d.C.), tal y como evidencian los entierros y numerosas muestras de cerámica en los estratos superiores del yacimiento. 


\section{Contexto regional: producción y consumo de la cerámica polícroma en la región cultural de Gran Coclé y territorios adyacentes}

Hallazgos previos desde inicios del siglo pasado, en la zona y época de estudio, muestran poca variabilidad regional de la producción de cerámica polícroma (festiva o ceremonial) en lo que se denomina región cultural de Gran Coclé (ver Figura 1). Los límites originales propuestos para dicha región abarcan ambas costas desde el río Tabasara hasta Chame y contamos con, al menos, tres zonas de producción formalmente propuestas: las llanuras coclesanas (Lothrop 1937, 1942), Veraguas (Lothrop 1948) y el Sur de Azuero (Ichon 1980). Éstas guardan gran parecido en cuanto a decoración y formas, siguiendo un gusto de época bastante uniforme, un mismo simbolismo y una misma tradición artística. Sin embargo desconocemos cómo se organizaba la producción de esta cerámica y como interactuaban los distintos grupos en esta región.

En la periferia cultural, llanuras próximas a la bahía de Chiriquí, se encontraron vasijas coclesanas de origen incierto (Holmes 1888; MacCurdy 1911). Sin embargo, su número es tan escaso que no se interpreta como un claro indicio de fluidas relaciones comerciales con las jefaturas al oeste de la frontera de la región cultural (Lothrop 1942). Las únicas semejanzas encontradas en excavaciones profesionales indican que hay cierto parecido con la vasijas coclesanas en la Fase San Lorenzo (Linares 1968) pero no del grupo polícromo sino de su estilo asociado Rojo sobre Crema, «red line» en Coclé (Lothrop 1942). En yacimientos de la bahía de Panamá, al este de los límites de Gran Coclé, se han reportado vasijas con indudables semejanzas con las reportadas en Coclé (Marshall 1949; Biese 1964; Cooke 1976b, 2001; Martín y Sánchez 2007) evidenciando que los contactos culturales eran más fluidos en esta región que hacia el este, con la zona de Chiriquí (Linares 1968).

Los datos que identifican la cerámica coclesana lejos de sus límites culturales, como sugieren ciertos autores (Reichel-Domatoff y Reichel-Domatoff 1961), son controvertidos, ya que no hay evidencia clara de interacción material como si ocurre dentro y en los límites de la misma. Es cierto que existe cierta similitud con algunas vasijas en el yacimiento de la costa del Pacífico colombiano de Cupica, sin embargo, no hay evidencia de cerámica polícroma pero sí de otros estilos asociados como la vajilla negro sobre blanco y rojo (panelled red en Coclé) y la ahumada (smoked ware), grupos cerámicos ampliamente extendidos entre las poblaciones amerindias, en especial con aquellas de lengua de ascendencia chibcha (Constella 1991) que habitaban una amplia zona desde Honduras hasta el sur de Colombia (Hoopes 2005: Fig. 1). También se ha mencionado la posibilidad de difusionismo coclesano en el yacimiento del Horno, en el Caribe colombiano (Reichel-Dolmatoff 1965). La única prueba son los diseños geométricos usados en la decoración de la cerámica coclé, de los cuales tenemos ejemplos en la cerámica encontrada en El Caño y que, en mi opinión, son sencillamente atributos decorativos compartidos que no evidencian por si solos contactos comerciales.

Por lo tanto, vemos que dentro de los límites establecidos para la región cultural de Coclé hay al menos 3 zonas de producción hipotéticas de cerámica polícroma que, en mi opinión, podrían ser simplemente dos por la uniformidad aparente de los contextos en Veraguas (Cooke y Ranere 1984; Cooke 2011): una situada en la cuenca de 
río Grande y otra en la cuenca del río Tonosí. La cerámica encontrada en Azuero, al este del Canajagua, en la bahía de Panamá (Marshall 1949) y en archipiélagos e islas (Linné 1929) guarda un asombroso parecido a la encontrada en Coclé y puede ser vista como producto de la interacción de los diferentes grupos que habitaban dentro de una misma región cultural y su periferia, donde la cerámica polícroma festiva y ritual era un producto de alto consumo en la demanda interna como un bien de prestigio en otros valles de la región (Haller 2004; Menzies 2009; Cooke 2011; Menzies y Haller 2012) y en yacimientos de la costa (Martín y Sánchez 2007). Estas cerámicas pudieron ser producidas en Coclé y ser comercializadas por otros bienes que no se encuentran en sus llanuras pero sí en su registro arqueológico, como metales, costillas de manatí (trichechidae), dientes fósiles de megalodonte (carcharodon megalodon), dientes de cachalote, basaltos o conchas para la manufactura de artesanías, joyería y herramientas.

Los datos cerámicos de yacimientos próximos a los límites culturales evidencian nítidos contactos con las regiones al este de los límites culturales y, en menor medida, con la región de Gran Chiriquí. Por el contrario, la muestra encontrada distante de los límites culturales, no es lo suficientemente clara como para determinar si estos fueron producto de intercambio. Sería necesario realizar infinidad de análisis de las composiciones químicas de las pastas para determinar la procedencia de las distintas vajillas, y más análisis estadísticos de variabilidad, como el planteado en este artículo, para determinar empíricamente el origen de estas piezas, sus zonas de producción y como se organizaban, así como conocer su consumo dentro y fuera de los límites culturales propuestos.

\section{Método}

Para determinar si una producción es estandarizada se suelen usar métodos arqueométricos con los cuales observamos el grado de parentesco entre las distintas vasijas (Rice 1981; Costin 1991; Longacre 1999). La homogeneidad de formas, decoración y características de la pasta, es un indicativo de estandarización en la producción y esto puede observarse en la variabilidad de la pigmentación de las pastas, en los colores empleados en la decoración, en los tamaños de las piezas, en la manipulación de desgrasantes y en la distribución geográfica, entre otros. Con ello nos podemos hacer una idea de cuáles eran sus técnicas de manufactura, si se empleaban las mismas técnicas para las diferentes vajillas, el grado de estandarización de las diferentes formas y diseños y el nivel de circulación y consumo del producto.

En este artículo, el análisis se basa exclusivamente en las formas y su variabilidad. La intención es observar la variabilidad existente en las formas del conjunto de muestras presumiblemente coetáneas, encontradas en una misma tumba y compararlas con datos etnográficos. Los problemas que nos encontramos a la hora de analizar muestras arqueológicas y compararlas con aquellas provenientes de los datos etnográficos es el factor temporal de los artefactos objetos de estudio. Los análisis de producción etnográficos suelen referirse a una misma unidad de producción plenamente contemporánea, mientras que normalmente los datos referentes a contextos arqueoló- 
gicos suelen proceder de diferentes unidades de producción no coetáneas. Estudios arqueométricos previos realizados en contextos urbanos en Siria (Blackman et al. 1993), revelan que los nexos entre estandarización y especialización artesanal pueden ser efectivos bajo condiciones espaciales y cronológicas similares. En El Caño encontramos depósitos funerarios colmatados, lo que permite abordar los análisis de producción de algunos grupos cerámicos como si estos fueran coetáneos, siendo aptos para compararlos con los datos etnográficos y evitar así los valores de «oscurecimiento temporal» ${ }^{1}$ (Blackman et al. 1993) que tienen otros estudios arqueológicos sobre estandarización, donde los valores resultantes deben ajustarse porcentualmente si los grupos cerámicos analizados pertenecen a eventos de producción no coetáneos (Costin y Hagstrum 1995; Balesta et al. 2009).

Se comprobará también la variabilidad de la cerámica no coetánea encontrada en los diferentes depósitos funerarios del yacimiento El Caño, pertenecientes a los dos periodos de ocupación del yacimiento. Estos se confrontarán con los resultados de la muestra coetánea, para conocer los valores de «oscurecimiento temporal» en la vajilla producida en Coclé, los cuales quizás puedan extrapolarse a otros estudios arqueológicos de sociedades con características socio-económicas y ambientales similares. Estos análisis se realizarán en ejemplos tanto de vajilla ritual o festiva como para la utilitaria, comparando si los valores de estandarización son los mismos o por el contrario se encuentran diferencias entre las distintas vajillas.

Como se ha mencionado con anterioridad, los análisis que se llevarán a cabo son los de coeficiente de variabilidad. Estos análisis determinan, en este caso, la oscilación de las medidas de las formas con respecto a la media del tamaño de todas las vasijas de la muestra. Es una unidad de medida utilizada en estudios de ciencias sociales (Longacre 1999; Clark 2007) y su ecuación es el resultado de la desviación típica $(\sigma)$ divida por el promedio (x) y multiplicado por 100:

$$
C_{v}=\frac{\sigma}{\bar{x}} \cdot 100
$$

Las medidas tomadas para el análisis fueron: el diámetro de boca, la altura y el diámetro máximo en el caso de los ejemplos de vasijas globulares o cuencos carenados. Estos grupos nos ayudarán a discernir los diferentes coeficientes de variabilidad tanto para vajillas polícromas (festivas o ceremoniales) como para vajillas de servicio o utilitarias, ya sean coetáneas o no. Al tener un gran número de muestras restauradas se optó por escoger los valores de éstas rechazando aquellas piezas incompletas, evitando así los errores de las mediciones de los fragmentos de borde que en ocasiones llega al $20 \%$ en producciones donde no se usa el torno. Los valores fuera de los rangos promedios, como algunas miniaturas o aquellas extraordinariamente grandes, cuyas dimensiones denotan diferentes usos al de los conjuntos seleccionados, fueron descartadas (Stark 1995). Estas no alcanzan a representar en ningún caso el 2\% de la muestra.

\footnotetext{
1 Los valores de coeficiente de variabilidad dentro de un mismo tipo cerámica aumentan considerablemente si estos no son coetáneos. Este aumento se denomina «oscurecimiento temporal» (Blackman et al. 1993).
} 


\section{Materiales}

Se analizaron 537 vasijas recuperadas en las excavaciones recientes del yacimiento de El Caño (Mayo y Mayo 2013), procedentes de cuatro tumbas y de los depósitos de ofrendas que tenían asociados, con una alta presencia tanto de vajilla ritual y festiva como de vajilla utilitaria. Fueron clasificadas en 12 categorías formales: platos, bandejas, cuencos, carafes ${ }^{2}$, ollas, efigies, miniaturas, soportes, incensarios y garrafas. Tanto para el análisis de variabilidad de las vasijas de las vajillas coetáneas como las no coetáneas, únicamente se tuvieron en cuenta aquellas formas de las categorías más numerosas. En el primer caso, las vajillas coetáneas, fueron platos (22), cuencos (8), carafes (8) y ollas (16). Para la vajilla no coetánea los platos (24), carafes (123) y ollas (66).

Los formas anteriormente descritas fueron adscritas a un grupo determinado siguiendo los criterios estilísticos empleados por Lothrop (1942) en la clasificación de las cerámicas de Sitio Conte. Para su clasificación, Lothrop tomó en consideración el número colores usados en la decoración de las vasijas y el orden de los mismos al ser aplicados. En El Caño se contabilizaron un total de 13 categorías de los 17 grupos descritos por Lothrop, de los cuales sólo son objeto de análisis aquellos más numerosos correspondientes al grupo Polícromo, al grupo Rojo (red ware) y al grupo Rojo sobre Crema (red line ware), las cuales comparten las formas descritas con anterioridad. Estos grupos ayudarán a discernir los diferentes valores de estandarización para las formas anteriormente mencionadas en los análisis de vajillas rituales y festivas (estilo Polícromo), así como de vajillas de servicio o utilitarias (estilo Rojo y estilo Rojo sobre Crema):

- Grupo Polícromo (ritual y festivo): es la vajilla más elaborada de todas las encontradas y su presencia representa el $34,26 \%$ del total de la muestra. Un engobe rojo claro cubre la totalidad de la pieza o sólo la cara interna. Se suele aplicar una gruesa capa $(0,05 \mathrm{~mm})$ de pigmento blanco, a modo de lienzo, antes de aplicar la decoración. En El Caño se recuperaron platos, cuencos, bandejas, carafes, garrafas y efigies (Figura 3). Se analizaron las formas de los platos que representan los dos periodos de ocupación del yacimiento.

- Grupo Rojo sobre Crema (utilitario): esta vajilla (red line en Lothrop 1942, Ladd 1964, o Guácimo en Cooke 1972) se caracteriza por ser una loza utilitaria muy común en contextos habitacionales y funerarios en Coclé y en el sur de la península de Azuero en su variedad Infiernillo Tacheté (Ichon 1980: 96). En El Caño se han encontrado ejemplos de ollas (Figura 4a) e incensarios (Figura $4 \mathrm{~b}$ ) que representan el $10,05 \%$ de la muestra. Estas se caracterizan por una decoración en rojo a base de manchas en forma de gotas y líneas sin el color negro usado de contorno típico de la vajilla polícroma. El tratamiento de la superficie varía entre alisados burdos y acabados pulidos que aparentan estar barnizados. La forma elegida para el análisis de esta vajilla son las ollas.

\footnotetext{
2 Se conoce como carafe a un contenedor sin asas, de cuerpo globular, cuello largo y bordes apuntados, rectos o redondeados. Fue usado probablemente para la ingesta de líquidos.
} 


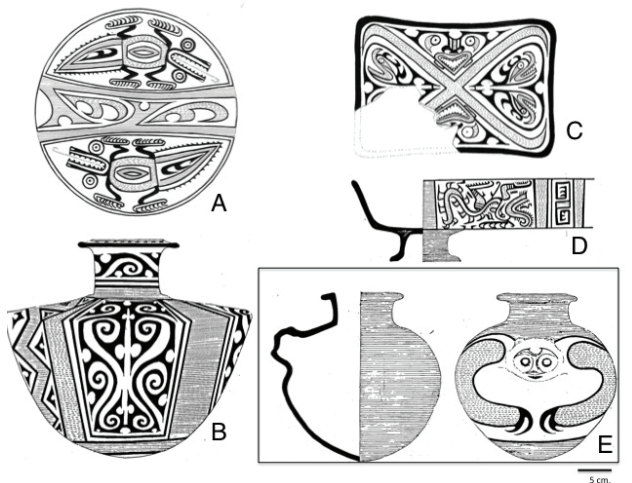

Figura 3: Cerámicas del Grupo Polícromo halladas en El Caño: a) plato; b) garrafa; c) bandeja; d) cuenco sobre pedestal; e) efigie zoomorfa (perezoso de dos dedos, Choloepus hoffmanni).
Figura 5: El Caño, ejemplares cerámicos del Grupo Rojo: a) garrafa; b) soporte; c) miniatura; d) carafe; e) plato de borde acanalado; f) cuenco; g) plato de borde engrosado; h) incensario; i) vasija efigie zoomorfa.

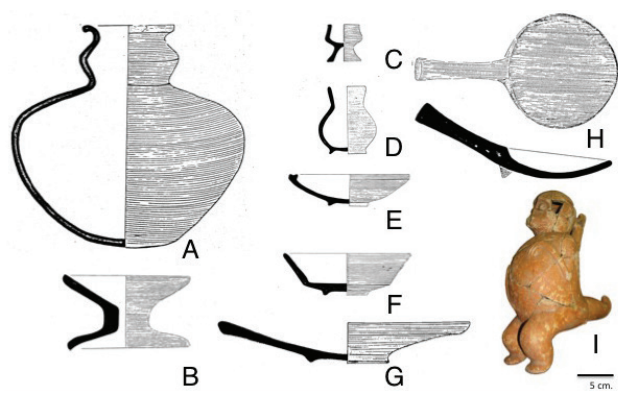

- Grupo Rojo (utilitario): es una cerámica utilitaria muy común tanto en zonas habitacionales como en yacimientos funerarios. En el Caño es la vajilla más numerosa, el 39,6 \% (Figura 5), siendo las formas más comunes los carafes, cuencos y platos, aunque también son habituales miniaturas, garrafas, soportes y las efigies. Se caracteriza por un fino engobe de color rojo que se aplica sobre la superficie de las piezas. Se eligió como material de análisis la forma de carafe al ser la representativa dentro de esta vajilla.figur

\section{Análisis}

\subsection{Coeficiente de variabilidad en platos, carafes y ollas no coetáneos}

Los grupos seleccionados para este análisis son platos polícromos (vajilla festiva y ritual), carafes rojos y ollas rojo sobre crema (vajilla utilitaria), pertenecientes a las diferentes tumbas excavadas con fechas de 780-1020 d.C. y de los depósitos estratigráficos asociados a estas. 

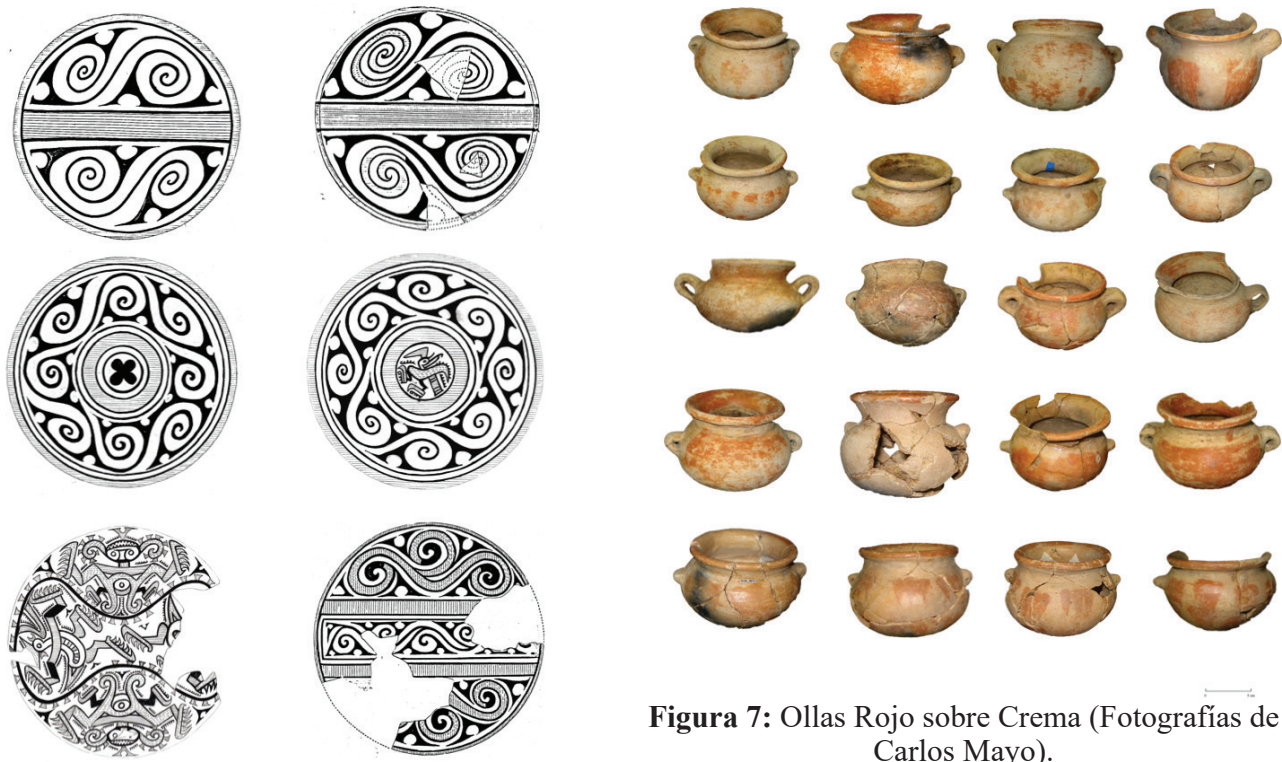

Figura 7: Ollas Rojo sobre Crema (Fotografías de Carlos Mayo).

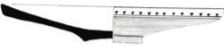

Figura 6: Platos polícromos encontrados en la Tumba 2 de El Caño.
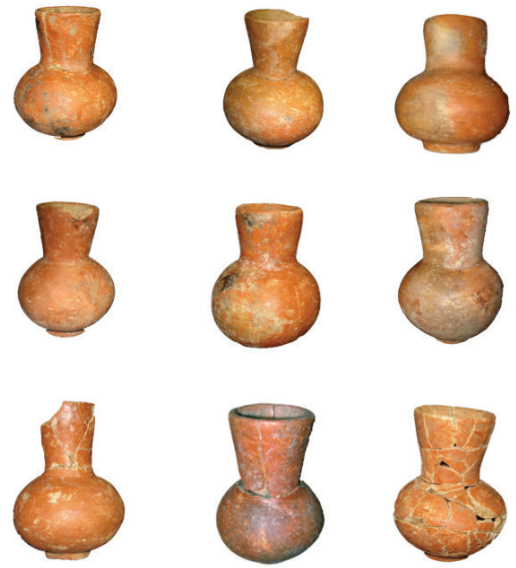

Figura 8: Carafes monocromos provenientes de la Tumba 2 de El Caño (Fotografías de Carlos Mayo).
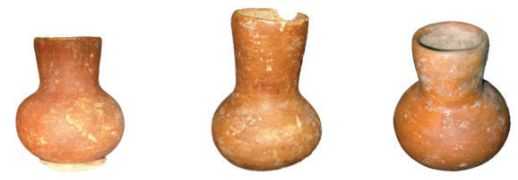

Los platos polícromos (Figura 6) son una forma muy común dentro de las tumbas. Se caracterizan por tener los bordes engrosados en el exterior en la mayoría de los casos $(72 \%)$ y en un número menor los que presentan bordes acanalados y sin modificar redondeados. Se tomaron las medidas de 24 platos, solo aquellos que presentaban el 
borde engrosado, con coeficientes de entre el 7,46\% del diámetro de boca y el 11,36\% de altura (Cuadro 1).

Se tomaron las medidas de las ollas (Figura 7), que se caracterizan por un cuerpo globular sin base, cuellos muy cortos con bordes evertidos y pequeñas asas de diferentes formas y tamaños. Las paredes suelen ser gruesas entre los 0,7 y los $1,2 \mathrm{~cm}$. y su color es rosado muy pálido (Munsell, Hue7.5 YR 8/2). Los desgrasantes son de color negro, rojo, blanco, claramente identificables por su tamaño mayor en ocasiones de $1 \mathrm{~mm}$. Los coeficientes de variabilidad de la muestra de 35 piezas arrojaron valores del $11,04 \%$ en el diámetro de boca, $11,15 \%$ en la altura y un $12,44 \%$ en el diámetro máximo de la panza (Cuadro 1).

La forma más numerosa encontrada en las tumbas son los carafes (Figura 8), la mayoría adscrita a la vajilla roja utilitaria. Su cuerpo es globular y se caracteriza por una base anular y un cuello largo con bordes rectos, redondeados o ligeramente apuntados. En ocasiones se le adjunta un asa vertedera, más común en las piezas polícromas. Los valores para este grupo adscrito a la vajilla roja son los más elevados de la muestras y se sitúan entre los $18,63 \%$ del diámetro máximo, el 15,43\% en el diámetro de la boca y el 19,27\% en la altura (Cuadro 1).

Cuadro 1: Coeficientes de variabilidad para cuencos, platos y carafes no coetáneos

\begin{tabular}{lcccc}
\hline \multicolumn{1}{c}{ Grupos cerámicos } & $\begin{array}{c}\text { Número de } \\
\text { piezas }\end{array}$ & $\begin{array}{c}\text { Diámetro } \\
\text { máximo } \mathbf{C}_{\mathbf{v}}\end{array}$ & $\begin{array}{c}\text { Diámetro de } \\
\text { boca } \mathbf{C}_{\mathbf{v}}\end{array}$ & $\begin{array}{c}\text { Altura } \\
\mathbf{C}_{\mathbf{v}}\end{array}$ \\
\hline Ollas rojo sobre crema (utlitaria) & 35 & $12,44 \%$ & $11,04 \%$ & $11,15 \%$ \\
Platos polícromos (festiva-ritual) & 24 & - & $7,46 \%$ & $11,36 \%$ \\
Carafes rojo (utilitaria) & 123 & $15,43 \%$ & $18,63 \%$ & $19,27 \%$ \\
\hline
\end{tabular}

\subsection{Coeficiente de variabilidad en platos y cuencos policromos presumiblemente coetáneos}

Para este análisis, además de los platos polícromos, los carafes rojos y las ollas rojo sobre crema seleccionados anteriormente, se observará la variabilidad de un grupo de cuencos polícromos. Estos análisis contemplan resultados para el periodo temprano y para el periodo tardío de ocupación del yacimiento:

Los cuencos (Figura 9) de la 3 fase de la Tumba 2 presentan homogeneidad en pasta, decoración y forma. Si a esto le sumamos que no tienen huellas de uso, sugieren que fueron manufacturados para el ritual funerario, como ofrenda, probablemente por una misma unidad de producción. La muestra es reducida ya que sólo contamos con 8 pequeños cuencos polícromos con decoración a modo de bandas alternas de color rojo, morado sobre el blanco del engobe. Son cuencos carenados con bordes convexos y los coeficientes de variabilidad son: $2,12 \%$ la altura, 2,28\% diámetro máximo y $2,74 \%$ el ancho de boca (Cuadro 2 ).

Los platos polícromos (22) elegidos para este análisis provienen de la Tumba 2 (14) y la Tumba 5 (8), cubriendo cuidadosamente los restos enterrados. Al igual que en el ejemplo de los cuencos no se observan huellas de uso y su marcado carácter ritual nos hace pensar que fueron manufacturados para el deceso y probablemente forman parte de una misma unidad de producción. Se eligieron los platos como 

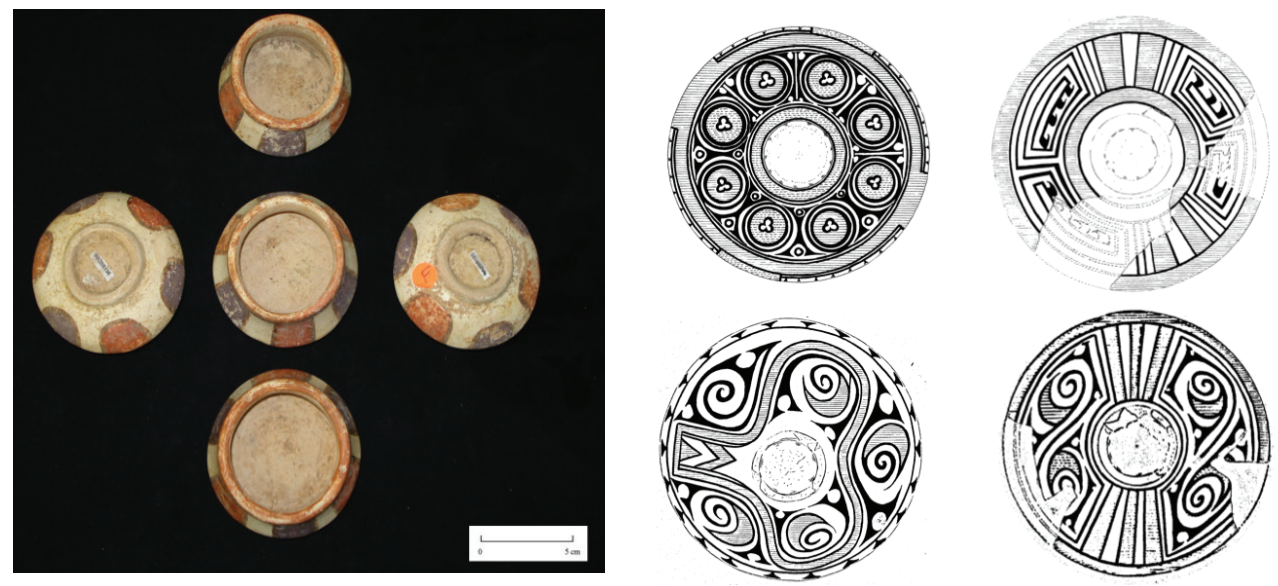

Figura 9: Cuencos polícromos proveniente de la Tumba 2 de El Caño (UE87) (Fotografías de Carlos Mayo).
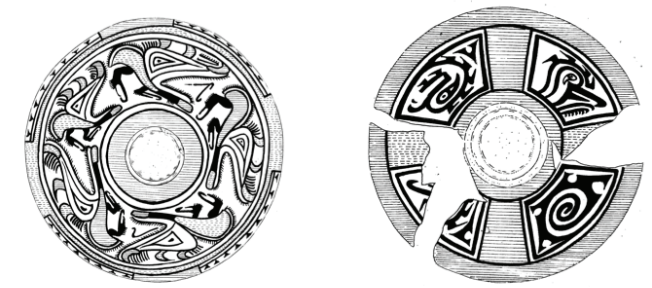

Figura 10: Platos pintados con decoración en el exterior provenientes de la Tumba 2 (UE 104) y probablemente coetáneos. Diámetros de 27,5 a $29 \mathrm{~cm}$.

segunda unidad de análisis para la vajilla polícroma al ser, en forma, de un tamaño considerablemente mayor al de los cuencos. Es relativamente más difícil tecnológicamente manufacturar objetos grandes que pequeños, sin embargo en nuestro ejemplo los valores no tienen variaciones significativas (Cuadro 2). Los valores de los platos de la Tumba 2 pertenecientes al periodo tardío son: diámetro 3,36\% y 3,98\% para la altura. Si tomamos una muestra del grupo y la reducimos tomando aquellos platos (8) que están decorados exclusivamente en el exterior (Figura 10) y que fueron probablemente manufacturados coetáneamente y por un mismo artesano, los valores son aun menores, observándose el 1,65\% para la boca y el 2,84\% para la altura. En la Tumba 5 , del periodo temprano, los valores son ligeramente más bajos a los obtenidos en la Tumba 2: $3,31 \%$ para el diámetro de la boca y 2,59\% para la altura (Cuadro 2).

En el caso de la cerámica utilitaria se eligieron por un lado los carafes, por ser la forma más numerosa dentro de la vajilla roja. Estos se encontraron en la unidad 88 de la Tumba 2 y son todos presumiblemente coetáneos. Los coeficientes en este caso son: $15,55 \%$ para el ancho de boca, $5,68 \%$ para la altura y $6,51 \%$ para el diámetro máximo del cuerpo (Cuadro 2). En un segundo análisis de vajilla utilitaria, la conocida como Red Line o Guacimo se analizaron las pequeñas ollas con porcentajes de coeficiente de variabilidad del $8,8 \%$ para el diámetro de boca, $11,9 \%$ para la altura y un $7,5 \%$ para el diámetro máximo del cuerpo (Cuadro 2). 
Cuadro 2: Coeficientes de variabilidad para cuencos, platos y carafes coetáneos

\begin{tabular}{lcrrrr}
\hline \multicolumn{1}{c}{ Grupos cerámicos } & $\begin{array}{c}\text { Tumba// } \\
\text { UE }\end{array}$ & $\begin{array}{c}\mathbf{N}^{\mathbf{o}} \text { de } \\
\text { piezas }\end{array}$ & $\begin{array}{c}\text { Diámetro } \\
\text { de boca } \\
\mathbf{C}_{\mathrm{v}}\end{array}$ & $\begin{array}{c}\text { Altura } \\
\mathbf{C}_{\mathrm{v}}\end{array}$ & $\begin{array}{c}\text { Diámetro } \\
\text { máximo } \\
\mathbf{C}_{\mathbf{v}}\end{array}$ \\
\hline Platos polícromos (festiva y ritual) & $2 / 104$ & 14 & $3,36 \%$ & $3,98 \%$ & - \\
Platos polícromos con decoración exterior (ritual) & $2 / 104$ & 8 & $1,65 \%$ & $2,84 \%$ & - \\
Platos polícromos (festiva y ritual) & $5 / 82$ & 8 & $3,31 \%$ & $2,59 \%$ & - \\
Cuencos polícromos (festiva y ritual) & $2 / 87$ & 8 & $2,74 \%$ & $2,12 \%$ & $2,28 \%$ \\
Carafes monocromos (utilitaria) & $2 / 88$ & 1 & $15,50 \%$ & $5,68 \%$ & $6,51 \%$ \\
Ollas (Red-Line) (utilitaria) & $5 / 82$ & 9 & $8,80 \%$ & $11,90 \%$ & $7,50 \%$ \\
\hline
\end{tabular}

\section{Análisis}

Los análisis de coeficiente de variabilidad de los grupos cerámicos analizados con anterioridad muestran diferentes grados de estandarización entre las diferentes vajillas coclesanas. Estos datos se compararan con los datos provenientes de las investigaciones etnográficas para inferir como se organizaba la producción de cerámica entre los coclé y cuál era su productividad.

En el registro etnográfico (Blackman et al. 1993; Longacre et al. 1988; Roux 2003) los coeficientes de variabilidad menores al 3\% indican una producción a gran escala, manufacturada en talleres por artesanos trabajando a tiempo completo con una productividad de unas 14.000 piezas por año y artesano (Roux 2003: Fig. 8). Los valores entre el $3 \%$ y el $6 \%$ se identifican con producciones de artesanos a tiempo completo con una productividad a gran escala, o bien, por artesanos a tiempo parcial con una productividad a pequeña escala cuya productividad estaría situada entre las 14.000 y 4.000 piezas por artesano al año (Roux 2003). Los valores superiores al 6\% se identifican con producciones a pequeña escala o muy baja escala en ambientes domésticos con artesanos trabajando a tiempo parcial con una productividad anual inferior a las 6.000 vasijas (Roux 2003). Sin embargo, estas estimaciones fueron realizadas para cerámica utilitaria hecha en torno, mientras que para la manufactura de los artefactos arqueológicos objeto de este estudio no se usó el torno. Para determinar la productividad de los artesanos en Coclé se aplicarán ajustes provenientes de datos etnográficos realizados en la península del Yucatán, México (Arnold et al. 2010) en la que se estima que un artesano con torno ahorra entre un 13\% y un $73 \%$ en el proceso de manufactura, con respecto a las producciones manuales.

Las cerámicas utilitarias coetáneas analizadas tienen resultados superiores al 6\% en los coeficientes de variabilidad, llegando en algunos casos hasta el 15\%. En el registro etnográfico, como mencionamos, estos valores se identifican con producciones de artesanos a tiempo parcial en ambientes domésticos, con una baja o muy baja productividad inferior a las 6000 vasijas anuales. Si aplicamos los ajustes derivados de los datos etnográficos para producciones con torno o manuales tendríamos que los artesanos de vajilla utilitaria en Coclé poseerían una productividad en torno a las 2.500 vasijas anuales.

El grupo polícromo coetáneo, el cual se interpreta como festivo y ritual, tiene coeficientes de variabilidad ligeramente superiores al 3\% e inferiores. Estos valo- 
res, comparados con los datos etnográficos, nos remiten a una producción altamente estandarizada concentrada en talleres, con una tasa de producción a gran escala y manufacturada por experimentados artesanos a tiempo completo. Sin embargo, esta organización del trabajo en talleres no existe como evidencia arqueológica en Coclé, por lo que sería temerario proponer dicho término en este trabajo. Además la cerámica objeto de estudio fue hecha con la técnica de enrollado y decorada, por lo que me parece improbable que un solo artesano haya podido tener una tasa tan alta de productividad. Si aplicamos el ajuste realizado por los estudios etnográficos de Arnold (2010) en Yucatán, tenemos una producción de cerámica por artesano de unas 6.000 piezas al año que, en mi opinión, pudo haber sido considerablemente más baja si la persona encargada de decorar las piezas fuera la misma que la encargada de su manufactura.

Por otro lado, los valores de coeficientes de variabilidad de las diferentes formas y vajillas encontradas en diferentes contextos no coetáneos son más altos si los comparamos con las formas coetáneas correspondientes presumiblemente en un mismo evento de producción. Esta diferencia estaría situada entre un $4 \%$ y un $8 \%$ para los valores de los platos del grupo polícromo. En las ollas rojo sobre crema los valores son superiores, entre el $2 \%$ y $4 \%$, mientras que en los carafes rojos son superiores al 10\%. La diferencia radica en que los depósitos arqueológicos excavados no son coetáneos. Estos datos quizás se podrían extrapolar a otros estudios arqueológicos sobre estandarización en los que solo se cuenta con muestras de cerámica no coetánea. Sin embargo, no hay una correspondencia clara entre los distintos grupos analizados. Observamos que las diferencias en el incremento de la variabilidad no se producen de una forma homogénea en las distintas unidades de análisis y existen marcadas diferencias en los valores de las dos vajillas utilitarias. Además vemos que los incrementos en los valores para los platos polícromos, la forma más estandarizada en las unidades de análisis coetáneas, son más elevados que para las ollas, una forma menos estandarizada en los análisis de los grupos coetáneos.

\section{Control económico de la producción de bienes en Coclé}

Tras los análisis de variabilidad, todo parece indicar que las jefaturas de Coclé se caracterizan por un sistema productivo especializado de cerámica polícroma. Queda ahora preguntarse si era una producción independiente o si, por el contrario, existía control por parte de una élite de este bien de consumo. La cerámica, a pesar de no ser un bien sobre el cual sea posible ejercer control de su producción, al existir materias primas para su manufactura disponibles en cualquier lugar y en grandes cantidades, ciertas piezas parecen tener un marcado carácter ritual o festivas: una manufactura sofisticada. Su acumulación en las tumbas más ricas y la nula o escasa presencia de ésta en los contextos funerarios humildes, dentro del propio yacimiento del Caño (Lleras y Barillas 1980) y otros en la cuenca de Río Grande (Lothrop 1942; Dade 1960), sugieren cierto control en su consumo.

En el yacimiento de El Caño, datos referentes a las excavaciones de los montículos evidenciaron entierros humildes donde las piezas polícromas son inexistentes o su 
presencia se reduce a pocos ejemplares (Lleras y Barillas 1980). Recientes excavaciones (Mayo y Mayo 2013) de personajes de alto rango dentro del yacimiento y coetáneos de los entierros humildes, nos ofrecen la oportunidad de comparar contextos y el consumo de cerámica entre las élites y el resto de la población. Las piezas polícromas en las que se emplean gran cantidad de recursos, tecnológicamente muy elaboradas y complejas, se acumulaban por decenas en los ritos funerarios de las personas poderosas. Sólo en la Tumba 2 del yacimiento se registraron un total de 118 vasijas polícromas en las tres fases de la tumba, frente a un máximo de 3 vasijas en los entierros de personas humildes situados, como se mencionó, en la zona de montículos. Es por tanto el número de las piezas polícromas y no la presencia de estas la que nos está marcando la riqueza en la tumba. Su consumo vemos que no es restringido pero su acumulación sugiere que fue producida por artesanos dependientes al servicio de una élite que controlaba la producción y consumo de esta cerámica con fines festivos, rituales y ceremoniales.

\section{Discusión}

En torno al año 700 se observan cambios políticos en la sociedad de los coclé que, según los expertos, pasan de sociedades tribales «igualitarias» a sociedades jerarquizadas o jefaturas (Linares 1977; Isaza 2007; Mayo y Mayo 2013). Si bien es cierto que parece existir consenso en cuando se produce el cambio, la discusión se centra en las causas. Se ha hecho mención (Redmond 1994) a los enfrentamientos entre grupos o clanes que posibilitaron la transformación a sociedades complejas, posiblemente motivados por el crecimiento de la población y la limitación de recursos. Estos enfrentamientos favorecieron el control sobre las mejores zonas de producción, de sus excedentes, de los bienes manufacturados y sus rutas de mercado, por parte de una élite que basaba su control en la fuerza institucionalizada (Linares 1977). Otro factor que pudo influir es el ideológico y el incremento del sistema simbólico que se manifiesta en la construcción de complejos funerarios como El Caño y en la riqueza iconográfica de sus esculturas (Mayo et al. 2010), cerámicas y orfebrería (Guinea y Mayo 2013). Estos posibilitaron una mayor expansión y unificación política en la región, legitimando el poder político con el ideológico y son un claro ejemplo de la influencia de estos poderes en la esfera económica. En este contexto económico, el proceso de producción especializado de bienes, con un marcado significado ritual y festivo, pudo actuar de forma determinante en el proceso formativo de esta jefatura.

En el registro arqueológico panameño, este proceso de producción especializado que se observa en la cerámica polícroma excavada en el Caño, sumado al control de su consumo y su valor comercial, sugieren que este pudo ser una de las claves en la génesis y esplendor de esta jefatura entre los siglos VIII-X d.C.

Los datos de estandarización sobre esta cerámica polícroma, comparados con el registro antropológico, sugieren producciones en talleres de artesanos cualificados trabajando a tiempo completo. Sin embargo, estos datos deben tomarse con cierta cautela a la hora de compararlos y al no haber evidencia arqueológica de talleres de cerámica en todo Panamá, me decanto por la posibilidad de producciones domésticas 
donde todos los miembros de un mismo ámbito familiar participaban en las tareas de decoración, manufactura, aprovisionamiento y tratamiento de las materias primas. Los datos arqueológicos de su consumo sugieren además cierta dependencia de la producción de esta cerámica por su valor comercial y ritual.

Por otro lado, la vajilla utilitaria ejemplificada en este trabajo en los grupos rojo y rojo sobre crema, indican valores bajos de estandarización que interpreto como producciones domésticas, probablemente elaboradas por artesanos a media jornada cuya productividad estaría situada en torno a las 2500 vasijas anuales. Estas producciones en el ámbito doméstico se complementaban probablemente con otras actividades económicas en las que, en mayor o menor medida, participaban todos los miembros de la familia. En Coclé probablemente esta manufactura se llevó a cabo principalmente en verano, lo que proporcionaría un óptimo secado de las pastas y eran lideradas posiblemente por las mujeres quienes se encargaban de la manufactura. Esta producción abastecería de piezas cerámicas el propio consumo de las unidades familiares y los excedentes podrían ser intercambiados localmente por otros productos.

Vemos por lo tanto diferentes tipos de organización de la producción en la cerámica de Coclé donde se repiten los modelos económicos de producción cerámica en Mesoamérica (Ball 1993). Estos modelos económicos revelan producciones de cerámica festiva y ritual, cuya manufactura era mayoritariamente consumida por una élite, junto a la manufactura de cerámica utilitaria producida en ambientes domésticos como complemento de las actividades desarrolladas en las economías familiares. Pero a priori no deben descartarse otros modelos derivados de análisis antropológicos y arqueológicos (Connell 2002), como las comunidades rurales de alfareros ubicadas cerca de las fuentes de materias primas y en zonas de agricultura marginal al observarse, en los diferentes grupos utilitarios y rituales provenientes de El Caño, las mismas formas, el uso de la mismas técnicas de manufactura y una aparente homogeneidad de las pastas.

AGRAdeCiMIENTOS: Esta investigación se ha llevado a cabo gracias al apoyo de la SENACYT (Secretaría de Ciencia y Tecnología de Panamá) y el INAC (Instituto nacional de Cultura de Panamá, Dirección de Patrimonio Histórico). Agradezco al STRI (Instituto Smithsonian de Investigaciones Tropicales) permitirme el uso de sus laboratorios en Ancón y su biblioteca. Estoy igualmente agradecido a Aurelio Sánchez por su esmero en la elaboración de los dibujos presentados en este artículo, a Elías Zaldivar, Rae Bauvient, Kim Coob y Ainslie Harrison por su capacitación y ayuda en la restauración del componente cerámico y a mi directora de tesis Josefa Rei y a Mercedes Guinea por la revisión de los borradores de este trabajo. Por último agradecer a la directora del proyecto Julia Mayo la confianza depositada en mí durante estos últimos años.

\section{Referencias bibliográficas}

Andagoya, Pascual de

1865 Narrative of the Proceedings of Pedrarias Davila. Londres: Hakluyt Society. 
Arnold, Dean E., Jill Hutar Willson y Antonio L. Nieves

2010 «Why Was the Potter's Wheel Rejected?: Social Choice and Technological Change in Ticul, Yucatán, Mexico», en Pottery Economics in Mesoamerica, Christopher A. Pool y George J. Bey, eds., pp. 59-87. Tucson: The University of Arizona Press.

BALL, Joseph W.

1993 «Pottery, Potters, Palaces, and Polities: Some Socioeconomic and PoliticaI lmplications of Late Classic Maya Ceramic Industries», en Lowland Maya Civilization in the Eighth Century A.D., Jeremy A. Sabloff y John S. Henderson, eds., pp. 243272. Washington: Dumbarton Oaks.

BALESTA Bárbara, Nora AZgorodny y Celeste VALENCiA

2009 «Evidencias de estandarización en la manufactura de cuencos de La Aguada, Orilla Norte (Argentina)». Estudios Atacameños 37: 79-98.

BENCO, Nancy L.

1988 «Morphological Standardization: An Approach to the Study of Craft Specialization», en A Pot for All Reasons: Ceramic Ecology, Charles C. Kolb y Louana M. Lackey, eds., pp. 57-72. Filadelfia: Laboratory of Anthropology, Temple University.

BIESE, Leo P.

1964 The Prehistory of Panama Viejo. Bureau of American Ethnology, Bulletin 191. Washington: Smithsonian Institution.

Blackman M. James, Gil J. Stein y Pamela B. Vandier

1993 «The Standardization Hypothesis and Ceramic Mass Production: Technological, Compositional, and Metric Indexes of Craft Specialization at Tell Leilan, Syria». American Antiquity 58 (1): 60-80.

CLARK, Geoffrey

2007 «Specialization, Standardization and Lapita Ceramics», en Oceanic Explorations: Lapita and Western Pacific Settlement, Stuart Bedford, Christophe Sand y Sean P. Connaughton, eds., pp. 289-299. Terra Australis 26. Canberra: The Australian National University E. Press.

Connell, Samuel V.

2002 «Getting Closer to the Source: Using Ethnoarchaeology to Find Ancient Pottery Making in the Naco Valley, Honduras». Latin American Antiquity 13 (4): 401-417.

Constella Umaña, Adolfo

1991 Las lenguas del Área Intermedia: introducción a su estudio areal. San José: Editorial de la Universidad de Costa Rica.

Cooke, Richard G.

1972 The Archaeology of the Western Coclé, Province of Panama. Tesis doctoral. Londres: University of London.

1976a «Rescate arqueológico en El Caño (NA-20), Coclé», en Actas del IV Simposium Nacional de Arqueología, Antropología y Etnohistoria de Panamá, pp. 447-482. Panamá: UNP/INAC.

$1976 b$ «Informe sobre excavaciones en el sitio CHO-3, Miraflores, río Bayano», en Actas del IV Simposium Nacional de Antropología, Arqueología y Etnohistoria de Panamá, pp. 369-426. Panamá: UNP/INAC.

1985 «Ancient Painted Pottery from Central Panama». Archaeology 38 (4): 33-39. 
2001 «Cupica (Choco): a Reassessment of Gerardo Reichel-Dolmatoff's Fieldwork in a Poorly Studied Region of the American Tropics», en Recent Advances in the Archaeology of the Northern Andes, A. Oyuela-Caycedo y J. Scott Raymond eds., pp. 91-106. Los Angeles: University of California.

2011 «The Gilcrease Collection and the Gran Cocle», en To Capture the Sun: The Gold from Ancient Panama, R. G. Cooke, N. J. Saunders, J. W. Hoopes y J. Quilter, eds., pp. 115-160. Tulsa: Gilcrease Museum.

Cooke, Richard G. y Anthony J. Ranere

1984 «The «Proyecto Santa Maria»: A Multidisciplinary Analysis of Prehistoric Adaptations to a Tropical Watershed in Panama», en Recent Developments in Isthmian Archaeology, F. W. Lange, ed., pp. 3-30. Oxford: BAR International Series 212.

Costin, Cathy Lynne

1991 «Craft Specialization: Issues in Defining, Documenting, and Explaining the Organization of Production». Archaeological Method and Theory 3: 1-56.

2000 «The Use of Ethnoarchaeology for the Archaeological Study of Ceramic Production». Journal of Archaeological Method and Theory 7 (4): 377-403.

2001 «Craft Production Systems», en Archaeology at the Millennium: A Sourcebook, Gary M. Feinman y T. Douglas Price, eds., pp. 273-327. Madison: University of Wisconsin.

Costin, Cathy Lynne y Melissa B. Hagstrum

1995 «Standardization, Labor Investment, Skill, and the Organization of Ceramic Production in Late Prehispanic Highland Peru». American Antiquity 60 (4): 619-639.

DADE, Philip L.

1960 «Rancho Sancho de la Isla: A Site in Coclé Province, Panama». Panama Archaeologist 3: 1 .

DoyLe, Gerald A.

1960 «Metal and Pottery Associations». Panama Archaeologist 3: 48-51.

Fernández De Oviedo VAldés, Gonzalo

1853 Historia Natural y General de Las Indias, Islas y Tierra Firma del Mar Océano, Vol. 3. Madrid: Real Academia de la Historia.

FitzGerald, Carlos

1992 «Informe preliminar sobre excavaciones arqueológicas en El Caño (NA-20), temporada 1988», en El Caño: Comunidad y Cultura, pp. 33-79. Panamá: Centro Subregional de Restauración OEA- INAC/Editorial Mariano Arosemena.

Guinea Bueno, Mercedes y Julia Mayo Torné

2013 «La armadura dorada de un cacique coclé: los pectorales de oro del ajuar del individuo principal de la Tumba 2 de la necrópolis de El Caño (900-1020 d.C.)». Revista Española de Antropología Americana 43 (2): 307-327.

Hagstrum, Melissa B.

1988 «Ceramic Production in the Central Andes, Peru: An Archaeological and Ethnographic Comparison», en A Pot for All Reasons: Ceramic Ecology, Charles C. Kolb y Louana M. Lackey, eds., pp. 127-145. Filadelfia: Laboratory of Anthropology, Temple University. 
HALLER, Mikael

2004 The Emergence and Development of Chiefly Societies in the Parita Valley, Panama. Tesis doctoral. University of Pittsburgh.

Helms, Mary W.

1995 Creations of the Rainbow Serpent: Polychrome Ceramic Designs from Ancient Panama. Albuquerque: University of New Mexico Press.

2000 The Curassow's Crest: Myths and Symbols in the Ceramics of Ancient Panama. Gainesville: University Press of Florida.

HoLmes, Willian Henry

1888 Ancient Art of the Province of Chiriqui, Colomiba (Panama). Washington: Smithsonian Institution.

Hoopes, John W.

2005 «The Emergence of Social Complexity in the Chibchan World of Southern Central America and Northern Colombia, AD 300-600». Journal of Archaeological Research 13 (1): 1-47.

ICHON, Alain.

1980 Archeologie du sud de la peninsula d'Azuero. Panama. México: Études Mésoaméricaines. Mission Archéologique et Ethnologique Française au Mexique, Serie II. CNRS.

IsAZA, Ilean

2007 The Ancestors of Parita: Pre-Columbian Settlement Patterns in the Lower La Villa River Valley, Azuero Peninsula, Panama. Tesis doctoral. Boston University.

KRAMER, Carol

1985 «Ceramic Ethnoarchaeology». Annual Review of Anthropology 14: 77-102.

LABBE, Armand

1995 Guardians of the Life Stream: Shamans, Art and Power in Pre-Hispanic Central Panama. Los Angeles: Cultural Art Press. The Bowers Museum of Cultural Art. The University of Washington Press.

LADD, John

1964 Archeological Investigations in the Parita and Santa Maria Zones of Panama. Washington D.C.: Smithsonian Institution, Bureau of American Ethnology, Bulletin 193.

LiNARES, Olga F.

1968 «Ceramic phases for Chirqui, Panama, and their relationship to neighboring sequences». American Antiquity 33 (2): 216-225.

1977 Ecology and the Arts in Ancient Panama: on the Development of Rank and Symbolism in the Central Provinces. Washington D.C.: Dumbarton Oaks Studies in Precolumbian Art and Archaeology 17.

LinNÉ, Sigvald.

1929 Darien in the past: the archaeology of eastern Panama and north-western Colombia. Göteborg: Elanders Boktryckeri Aktiebolag.

Lleras PÉREZ, Roberto y Ernesto BARILlas CORDÓN

1980 Excavaciones Arqueológicas en el Montículo 4 de El Caño. Panamá: Instituto Nacional de Cultura y Centro de Restauración OEA- INAC. 
LONGACRE, William

1999 «Standardization and Specialization: What's the Link?», en Pottery and People, J. M. Skibo y G. M. Feinman, eds., pp. 44-58. Salt Lake City: University of Utah Press.

LONGaCre, William, Kenneth L. Kvamme y Masashi KobaYASHY

1988 «Southwestern Pottery Standardization: An Ethnoarchaeological View from the Philippines». The Kiva 53 (2): 101-112.

Lothrop, Samuel K.

1937 Coclé: An Archaeological Study of Central Panama, Part 1. Cambridge: Memoirs of the Peabody Museum of Archaeology and Ethnology 7. Harvard University Press.

1942 Coclé: An Archaeological Study of Central Panama, Part 2. Cambridge: Memoirs of the Peabody Museum of Archaeology and Ethnology 8. Harvard University Press.

1948 Archaeology of Southern Veraguas, Panama. Cambridge: Memoirs Peabody Museum of Archaeology and Ethnology 9 (3). Harvard University.

1976 Pre-Columbian Design from Panama. Nueva York: Dover Publications.

MACCURDY, George G.

1911 A Study of Chiriquian Antiquities. New Haven: Memoirs of the Connecticut Academy of Arts and Sciences Vol. 3. Yale University Press.

Marshall, Donald S.

1949 «Archaeology of Far Fan Beach, Panama Canal Zone». American Antiquity 15 (2): 124-132.

MARTin, Juan Guillermo y Luís Alberto SÁNCHEZ

2007 «El istmo mediterráneo: intercambio, simbolismo y filiación social en la bahía de Panamá durante el período 500-1000 DC». Arqueología del Área Intermedia 7: 113-121.

MAYO TORnÉ, Julia

2006 «Los estilos cerámicos de la región cultural de Gran Coclé, Panamá». Revista Española de Antropología Americana 36 (1): 25-44.

MAYO Torné, Julia y Carlos MAYo TORné

2013 «El descubrimiento de un cementerio de élite en El Caño: indicios de un patrón funerario en el valle de Río Grande, Coclé, Panamá». Arqueología Iberoamericana 20: $3-27$.

MaYo Torné, Julia, Carlos MaYo Torné y Vicky Karas

2010 «La escultura precolombina del área intermedia. Aproximación al estudio estilístico, iconográfico y espacial del grupo escultórico de El Caño», en Producción de bienes de prestigio ornamentales y votivos de la América antigua, Emiliano Melgar, Reyna Solís y Ernesto González, compiladores, pp. 85-96. Deale: Syllaba Press.

MenzIES, Adam C.J.

2009 Craft Specialization and the Emergence of the Chiefly Central Plate Community of HE-4 (El Hatillo), Central Panamá. Ph.D. Dissertation. Universidad de Pittsburgh. 
Menzies, Adam C.J. y Mikael J. Haller

2012 «A Macroregional Perspective on Chiefly Cycling in the Central Region of Panama during the Late Ceramic II Period (A.D. 700-1522)». Latin American Antiquity 23 (4): 449-466.

Mojica, Alexis, Julia Mayo, Carlos Mayo, José Ramón Chantada e Itzel De Gracia

2007 «Resultado de las prospecciones magnética y eléctrica del yacimiento arqueológico El Caño (NA-20), Gran Coclé, Panamá.» Revista Española de Antropología Americana 37(1): 111-126.

REDMOND, Elsa M.

1994 «External warfare and the internal politics of northern South American tribes and chiefdoms», en Fractional Competition and Political Development in the New World, Elizabeth M. Brumfiel y John W. Fox, eds., pp. 44-54. Cambridge: Cambridge University Press.

Reichel-DolmatofF, Gerardo

1965 Colombia. Ancient Peoples and Places Series 44. Londres: Thames and Hudson.

Reichel-Dolmatoff, Gerardo y Alicia Reichel-DolmatofF

1961 «Investigaciones arqueológicas en la costa Pacífica de Colombia: El sito de Cupica». Revista Colombiana de Antropología 10: 239-317.

RICE, Prudence M.

1981 «Evolution of Specialized Pottery Production: A Trial Model». Current Anthropo$\log y 22$ (3): 219-240.

1991 «Specialization, Standardization, and Diversity: A Retrospective», en The ceramic legacy of Anna Shepard, Ronald L. Bishop y Frederick W. Lange, eds., pp. 257279. Niwot: Colorado University Press.

Roux, Valentine

2003 «Ceramic Standardization and Intensity of Production: Quantifying Degrees of Specialization». American Antiquity 68 (4): 768-782.

STARK, Barbara L.

1995 «Problems in Analysis of Standardization and Specialization in Pottery», en Ceramic Production in the American Southwest, Barbara J. Mills y Patricia L. Crown, eds., pp. 231-268. Tucson: The University of Arizona Press.

VerriLl, Alpheus H.

1927 «A Mystery of the Vanished Past in Panama: Newly Discovered Relics of a Vanished Civilization Destroyed by Earthquake or Volcanic Eruption». Illustrated London News 173: 15-18.

ZELSMAN, J.

1959 «A Río Grande Burial». Panama Archaeologist 2: 85-90. 